\title{
Familial hypercholesterolaemia with cardiovascular disease:a case report
}

\author{
Kexin Wang ${ }^{1}$, Tao Sun ${ }^{1}$, Xiaoping Zhang ${ }^{2}$, Hai Gao ${ }^{1}$, and Xiaoyan $\mathrm{Li}^{2}$ \\ ${ }^{1}$ Beijing An Zhen Hospital \\ ${ }^{2}$ Beijing Institute of Heart Lung and Blood Vessel Diseases
}

September 25, 2021

\begin{abstract}
A 56-year-old female with definite FH was reported based on clinical algorithms. Whole exome sequencing identified a heterozygous LDLR mutation (c.1599G>A), which is pathogenic according to ACMG guidelines. Sanger sequencing was performed in family members, and the mutation site was co-segregated with the disease in the family.
\end{abstract}

\section{Introduction}

Familial hypercholesterolemia $(\mathrm{FH})$ has been identified as an autosomal dominant disorder and is a major contributor to premature atherosclerotic cardiovascular disease (ASCVD). Mutations in four genes have been reported to be responsible for an estimated 70-95\% of FH: LDLR, Apo B, PCSK9, and LDLRAP. Additionally, LDLR mutation has been the most common cause. (1-4) This case reported a 56-year-old female with unstable angina and elevated plasma LDL-C level, carried a heterozygous pathogenic LDLR mutation (c.1599G >A), the patient was a definite FH according to DLCN criteria. The sanger sequencing on the family members confirmed 3 members carried a heterozygous mutation in the previous site, and the clinical investigations were consistent with the sequencing.

\section{Case presentation}

A 56-year-old female with unstable angina was performed percutaneous coronary intervention (PCI). The right coronary artery (RCA) was diffuse from the proximal segment to the distal segment, the proportion of three parts of the right coronary artery were $75 \%, 99 \%$ and $60 \%$, respectively. 1 stent was placed in the RCA. (Figure 1) Aspirin (100mg/day), clopidogrel (75mg/day), rosuvastatin (20mg/day), metoprolol $(47.5 \mathrm{mg} /$ day), and isosorbide mononitrate $(40 \mathrm{mg} /$ day) were used after the intervention. After one year, the medications were adjusted to aspirin $(100 \mathrm{mg} /$ day $)$, rosuvastatin $(20 \mathrm{mg} /$ day $)$, ezetimibe $(10 \mathrm{mg} /$ day $)$, and bisoprolol fumarate $(10 \mathrm{mg} /$ day $)$.

The patient had been found a high level of blood lipid at the age of 35(Table1), but received treatment irregularly and the particular method was unknow. Without other conditions that could lead to secondary hypercholesterolemia or phytosterolemia. Whole exome sequencing was performed, and one candidate mutation was identified: LDLR (NM_000527.4): c. 1599G >A (p. Trp533*) (Figure 3). This mutation was pathogenic according to ACMG guidelines. Based on the patient's medical history, early on-set ASCVD family history and information of sequencing, a definite FH could be evaluated according to DLCN criteria.

Clinical investigation indicated that family members II1 had a high lipid level and ASCVD at the age of 56, II2 and III 2 were found to have elevated LDL-C at the age of 51 and 41, respectively, but have no ASCVD yet (Table1). The sanger sequencing for 3 family members (II1, II2, III2) certified the heterozygous mutation. While III3 and IV1 were wild type. 
Expect for family member III2, the other family members were treated with statins regularly, and adjusted to statins and ezetimibe combined lipid-lowering therapy after the effect was poor. So far, no recurrence or occurrence of cardiovascular or cerebrovascular events have been reported.

\section{Discussion}

The most common gene mutation that causes FH is LDLR. More than 3000 LDLR mutations have been reported in ClinVar. (4-6) There are many clinical criteria for FH, but genetic testing is still the gold standard for diagnosis (7). In this case, a heterozygous LDLR mutation (c. 1599G>A) was identified by whole exome sequencing and sanger sequencing for family members.

In this case, family members with heterozygous LDLR mutation have LDL-C level fluctuated between 5$7.9 \mathrm{mmol} / \mathrm{L}$ which can be simply judged as heterozygous FH from the LDL-C level[?]190 $\mathrm{mg} / \mathrm{dL}$ (5 mmol/L) in an adult. (7) However, family members' DLCN score classified as probable FH (score 7-8) before genetic sequencing. Positive sequencing results clarified their diagnoses were definite $\mathrm{FH}$, and improved the efficiency of this cascade screening to some extent.

Early and appropriate lipid-lowering therapy is essential for patients with FH and ASCVD $(8,9)$. The index patient was irregularly used statin at age 35, and the reduction was unknown. After PCI and genetic sequencing, reduction in patient who used statins and ezetimibe in combination with lipid-lowering during a year was 55.2\%-62.7\%. Therefore, FH with heterozygous LDLR mutation (c. 1599G >A) may be suitable for combination lipid-lowering therapy with statins and ezetimibe. In addition, the family members(II1, II2 and III2) used statins at the early stage. However, the reduction was $20.5 \%$ (II1), $15.1 \%$ (II2) and $13.3 \%$ (III2), the treatment adjusted to the combined lipid-lowering therapy of statins $(20 \mathrm{mg} /$ day $)$ and ezetimibe (10mg/day) after cascade screening. For family member III2, it is strongly suggested to strengthen lipid-lowering therapy, improve bad living habits, and further coronary CTA or coronary angiography can be carried out. After sequencing, family member III2 did reexamination that the LDL-C level is $4.12 \mathrm{mmol} / \mathrm{L}$, the reduction is $47.8 \%$. It was found that positive genetic confirmation of $\mathrm{FH}$ recognizes the importance, increases confidence in the role of lipid-lowering medicines, motivates initiation and adherence to lipid-lowering treatments.

In conclusion, comparing with traditional screening, cascade screening is more cost-effective. Genetic testing and cascade screening improve cognition and treatment adherence in $\mathrm{FH}$ patients. Besides, genetic testing to somewhat enhance the efficiency of cascade screening.

\section{Acknowledgements}

We thank the patient and her family members for their interest and cooperation.

Funding: The study was supported by the National Natural Science Foundation of China (81400846).

\section{Footnote}

Conflicts of Interest: All authors have completed the ICMJE uniform disclosure form and declare: The authors have no conflicts of interest to declare.

Ethical Statement: The authors are accountable for all aspects of the work in ensuring that questions related to the accuracy or integrity of any part of the work are appropriately investigated and resolved. All procedures performed in studies involving human participants were in accordance with the Helsinki Declaration (as revised in 2013), and this study was approved by the ethics committee of the Beijing Anzhen Hospital of Capital Medical University. Written informed consent was obtained from the patient for publication of this Case report and any accompanying images. A copy of the written consent is available for review by the Editor-in-Chief of this journal.

Open Access Statement: This is an Open Access article distributed in accordance with the Creative Commons Attribution-NonCommercial-NoDerivs 4.0 International License (CC BY-NC-ND 4.0), which permits the non-commercial replication and distribution of the article with the strict proviso that no changes or edits 
are made and the original work is properly cited (including links to both the formal publication through the relevant DOI and the license). See:https://creativecommons.org/licenses/by-nc-nd/4.0/.

Author contributions: Wang KX participated in collecting the data and drafted the manuscript; Sun T and Zhang XP carried out the studies, performed the genetic analysis; Gao H, and Li XY Been involved in drafting the manuscript or revising it critically for important intellectual content and the acquisition of funding, general administrative support. All authors have read and approved the final version of the manuscript. all authors have read and approved the final version of the manuscript.

\section{References}

1. Benito-Vicente A, Uribe KB, Jebari S, Galicia-Garcia U, Ostolaza H, Martin C. Familial Hypercholesterolemia: The Most Frequent Cholesterol Metabolism Disorder Caused Disease. Int J Mol Sci. 2018 Nov 1;19(11):3426. doi: 10.3390/ijms19113426. PMID: 30388787; PMCID: PMC6275065.

2. Watts GF, Gidding SS, Mata P, Pang J, Sullivan DR, Yamashita S, Raal FJ, Santos RD, Ray KK. Familial hypercholesterolaemia: evolving knowledge for designing adaptive models of care. Nat Rev Cardiol. 2020 Jun;17(6):360-377. doi: 10.1038/s41569-019-0325-8. Epub 2020 Jan 23. PMID: 31974482.

3. Defesche JC, Gidding SS, Harada-Shiba M, Hegele RA, Santos RD, Wierzbicki AS. Familial hypercholesterolaemia. Nat Rev Dis Primers. 2017 Dec 7; 3:17093. doi: 10.1038/nrdp.2017.93. PMID: 29219151.

4. Gidding SS, Champagne MA, de Ferranti SD, et al. The agenda for familial hypercholesterolemia: a scientific statement from the American Heart Association. Circulation. 2015 Dec 1;132(22):216792. doi: 10.1161/CIR.0000000000000297. Epub 2015 Oct 28. Erratum in: Circulation. 2015 Dec 22;132(25): e397. PMID: 26510694.

5. Iacocca MA, Chora JR, Carrié A, et al. ClinV ar database of global familial hypercholesterolemiaassociated DNA variants. Hum Mutat. 2018 Nov;39(11):1631-1640. doi: 10.1002/humu.23634. PMID: 30311388; PMCID: PMC6206854.

6. Wang J, Dron JS, Ban MR, et al. Polygenic Versus Monogenic Causes of Hypercholesterolemia Ascertained Clinically. Arterioscler Thromb Vasc Biol. 2016 Dec;36(12):2439-2445. doi: 10.1161/ATVBAHA.116.308027. Epub 2016 Oct 20. PMID: 27765764.

7. Moldovan V, Banescu C, Dobreanu M. Molecular diagnosis methods in familial hypercholesterolemia. Anatol J Cardiol. 2020 Feb;23(3):120-127. doi: 10.14744/AnatolJCardiol.2019.95038. PMID: 32120369; PMCID: PMC7222642.

8. Raal FJ, Hovingh GK, Catapano AL. Familial hypercholesterolemia treatments: Guidelines and new therapies. Atherosclerosis. 2018 Oct; 277:483-492. doi: 10.1016/j.atherosclerosis.2018.06.859. PMID: 30270089.

9. Lee S, Akioyamen LE, Aljenedil S, et al. Genetic testing for familial hypercholesterolemia: Impact on diagnosis, treatment and cardiovascular risk. Eur J Prev Cardiol. 2019 Aug;26(12):1262-1270. doi: 10.1177/2047487319829746. Epub 2019 Feb 12. PMID: 30755017.

10. Hardcastle SJ, Legge E, Laundy CS, Egan SJ, French R, Watts GF, Hagger MS. Patients' perceptions and experiences of familial hypercholesterolemia, cascade genetic screening and treatment. Int J Behav Med. 2015 Feb;22(1):92-100. doi: 10.1007/s12529-014-9402-x. PMID: 24585182.

Figure 1 Schematic diagram of coronary angiography of the patient. Blue marker: target lesion; LMA (left main artery) was irregular, LAD (left anterior artery) with $75 \%$ tubular stenosis in the middle segment; no significant stenosis of LCX (left circumflex artery); the proportion of three parts of RCA (right coronary artery) were $75 \%, 99 \%$ and $60 \%$.

Figure 2 Pedigree of the patient. II4 was diagnosed as FH and ASCVD. Cascade screening for family members (II1, II2, III2, III3 and IV1) found that II1, II2 and III2 was FH, II1 was diagnosed as CAD and received the PCI.

Figure 3 Sanger sequencing for the candidate mutation of the family members. Whole exome sequencing for the index patient identified a mutation: LDLR (NM_000527.4): c. 1599G >A (p. Trp533*). The sanger 
sequencing for family members, III, II2 and III2 were found the heterozygous mutation in the candidate site. IV1 were wild type.

\begin{tabular}{lccccc}
\hline & $\begin{array}{c}\text { Sex } \\
\text { M/F }\end{array}$ & $\begin{array}{c}\text { Age/LDL-C } \\
(\mathrm{mmol} / \mathrm{L})\end{array}$ & $\begin{array}{c}\text { Age/LDL-C } \\
(\mathrm{mmol} / \mathrm{L})\end{array}$ & Age of ASCVD & DLCN \\
\hline Index patient (II4) & $\mathrm{F}$ & $35 \mathrm{y} / 5-6$ & $58 \mathrm{y} / 2.24$ & $56 \mathrm{y}$ & Definite FH \\
The eldest sister (III) & $\mathrm{F}$ & $56 \mathrm{y} / 6.06$ & $67 \mathrm{y} / 3.37$ & $56 \mathrm{y}$ & Definite FH \\
The second elder sister (III) & $\mathrm{F}$ & $51 \mathrm{y} / 7.19$ & $58 \mathrm{y} / 6.25$ & - & Definite FH \\
Nephew (III2) & $\mathrm{M}$ & $39 \mathrm{y} / 7.90$ & $42 \mathrm{y} / 6.85$ & - & Definite FH \\
Daughter (III3) & F & $31 \mathrm{y} /$ normal & - & - & Unlikely FH \\
\hline
\end{tabular}

Table 1 Serum LDL-cholesterol level and diagnosis of the family.

F, female; M, male; LDL-C, low density lipoprotein cholesterol; ASCVD, arteriosclerotic cardiovascular disease; DLCN, Dutch Lipid Clinical Network criteria.
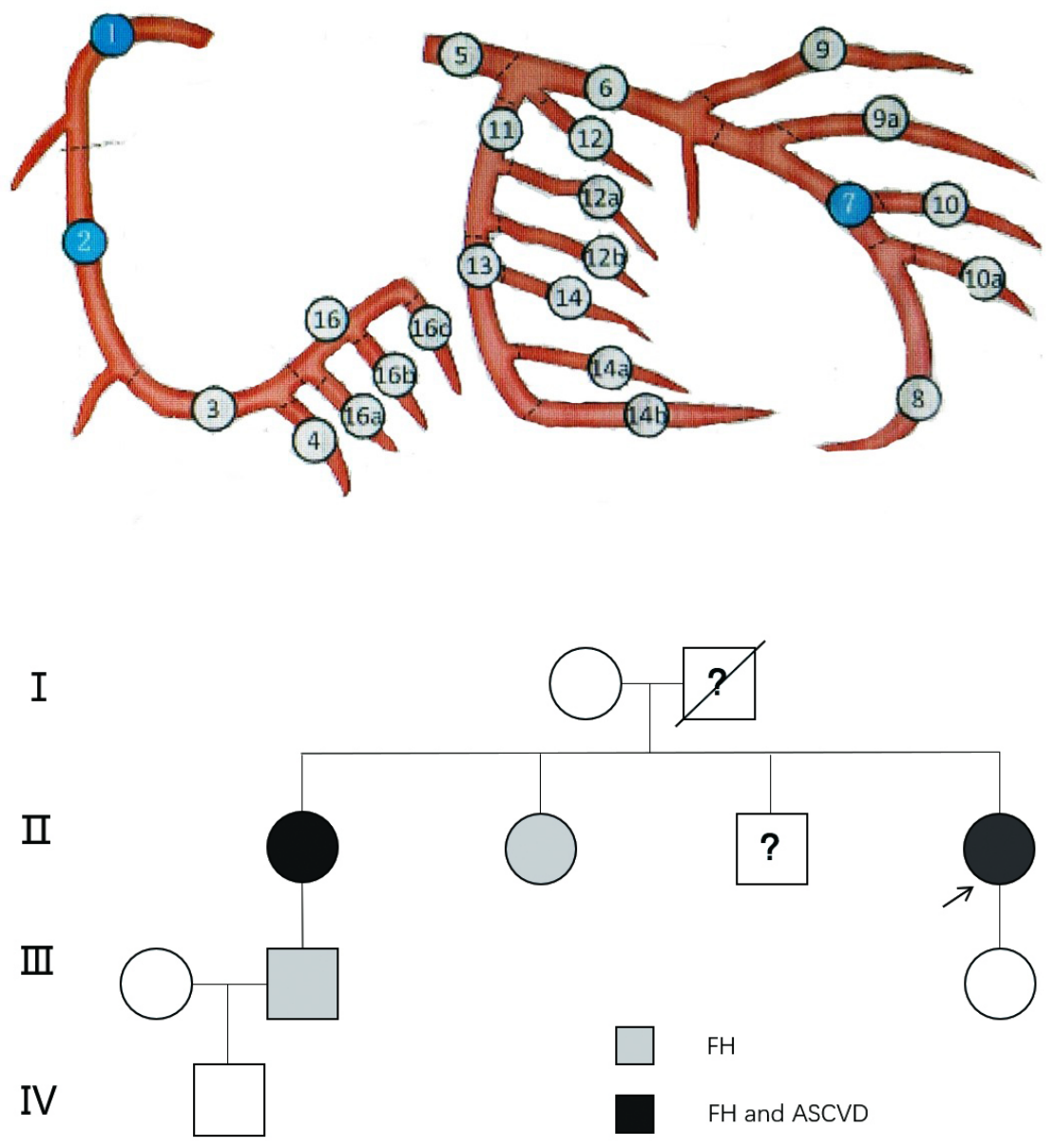


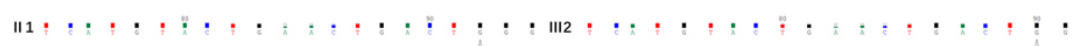
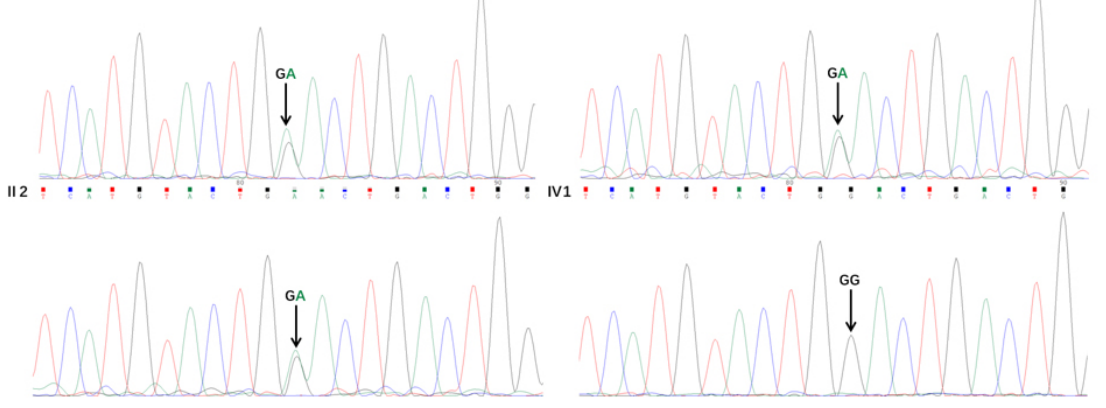